\title{
Motivic Zeta Function via Dlt Modification
}

\section{Chenyang Xu}

\begin{abstract}
AвSTRACT. Given a smooth variety $X$ and a regular function $f$ on it, by considering its dlt modification, we define the dlt motivic zeta function $Z_{\text {mot }}^{\mathrm{dlt}}(s)$ which does not depend on the choice of the dlt modification.
\end{abstract}

\section{Introduction}

Let $k$ be a field of characteristic 0 . Kontsevich invented the concept of motivic integration, which upgrades the $p$-adic integration with the value in a modification of the Grothendieck ring $K_{0}\left(\operatorname{Var}_{k}\right)$. One main application of motivic integration is to the definition and study of the motivic zeta function (see [DL98]), which is a corresponding upgrade of Igusa's $p$-adic zeta function. More precisely, let $X$ be a smooth $k$-variety of pure dimension, and let $D=V(f)$ be the zero divisor for a $k$-morphism to the affine line. We define the (naive) motivic zeta function $Z_{\text {mot }}(f, s)$ (or abbreviated as $\left.Z_{\text {mot }}(s)\right)$ of $(X, D)$ by

$$
Z_{\text {mot }}(s)=\int_{\mathcal{L}(X)} \mathbb{L}^{-\left(\operatorname{ord}_{t} D\right) s} \in \mathcal{M}_{k}\left[\left[\mathbb{L}^{-s}\right]\right] .
$$

Here $\mathcal{L}(X)$ is the arc space of $X \operatorname{ord}_{t}(D)$ is the function $\mathcal{L}(X) \rightarrow \mathbb{N} \cup\{\infty\}$ associated to the vanishing order of the arc along the divisor $D$, and $\mathcal{M}_{k}=$ $K_{0}\left(\operatorname{Var}_{k}\right)\left(\mathbb{L}^{-1}\right)$ where $\mathbb{L}=\left[\mathbb{A}_{k}^{1}\right]$.

In [DL98], an explicit formula for the motivic zeta function, using a log resolution of $(X, D)$ is given as follows. Let $h: Y \rightarrow(X, D)$ be a $\log$ resolution that is an isomorphism over $X \backslash D$. Denote by $E_{i}(i \in I)$ the irreducible components of the divisor $E=h^{-1}(D)$, and by $\left(N_{i}, v_{i}\right)$ the corresponding pair

$$
\text { (mult } \left._{E_{i}}\left(h^{*} D\right), a\left(E_{i}, X\right)+1\right),
$$

where $a\left(E_{i}, X\right)$ is the discrepancy. For any nonempty subset $J$ of $I$, we put $E_{J}=$ $\bigcap_{i \in J} E_{i}$ and $E_{J}^{0}=E_{J} \backslash \bigcup_{i \notin J} E_{i}$. We denote by $d$ the dimension of $X$. With this notation, we have

$$
Z_{\mathrm{mot}}(s)=\mathbb{L}^{-d} \sum_{J \subset I}\left[E_{J}^{0}\right] \prod_{i \in J} \frac{(\mathbb{L}-1) \mathbb{L}^{-N_{i} s-v_{i}}}{1-\mathbb{L}^{-N_{i} s-v_{i}}} \in \mathcal{M}_{k}\left[\left[\mathbb{L}^{-s}\right]\right] .
$$

It is clear that the candidates of the poles are of the form $s=-v_{i} / N_{i}$. (We note that $\mathcal{M}_{k}$ is not a domain, so for the precise meaning of a pole, see Remark 2.1.) However, many of them will cancel out. Thus, determining the poles is a challenging problem. In fact, the famous monodromy conjecture predicts that any pole

Received August 22, 2014. Revision received February 10, 2015.

The author is partially supported by the grants "The Recruitment Program of Global Experts" and

"The Chinese National Science Fund for Distinguished Young Scholars". 
$s$ of the motivic zeta function is a root of the Berstein-Sato polynomial $b_{s}(f)$. A weaker version predicts that $e^{2 \pi i s}$ is an eigenvalue of the local monodromy action on the cohomology of the Milnor fiber of $f$ at some point $x \in D=V(f)$.

In this paper, by looking at the divisorial log terminal (dlt) modification of the pair $\left(X, D_{\text {red }}\right)$ we define an alternative zeta function with coefficients in a finite extension of $\mathcal{M}_{k}$. We recall that a dlt modification is an (often nonunique) partial resolution $\left(X^{\mathrm{dlt}}, D^{\mathrm{dlt}}\right) \rightarrow(X, D)$, which is introduced in the minimal model program (MMP) theory, and turns out to be very useful in the study of singularities of pairs.

In fact, for a $\log$ canonical pair $(X, \Delta)$, an effective $\mathbb{Q}$-divisor $M$ on $X$ and any dlt pair $\left(X^{\mathrm{dlt}}, D^{\mathrm{dlt}}\right)$, which admits a birational morphism $g: X^{\mathrm{dlt}} \rightarrow X$, we will define an associated motivic zeta function. More precisely, we consider the stratification of $\left(X^{\mathrm{dlt}}, D^{\mathrm{dlt}}\right)$ by its $\log$ canonical centers and write $\left\lfloor D^{\mathrm{dlt}}\right\rfloor=\sum E_{i}$ $(i \in I)$ for the sum of reduced divisors. For any $J \subset I$, we can similarly define $E_{J}^{0}=\bigcap_{j \in J} E_{j} \backslash\left(\bigcup_{i \notin J} E_{i}\right)$ as the union of open strata and write

$$
\left.\left(K_{X^{\mathrm{dlt}}}+D^{\mathrm{dlt}}\right)\right|_{E_{J}^{0}}=K_{E_{J}^{0}}+D_{J}^{0} .
$$

In particular, we know that $\left(E_{J}^{0}, D_{J}^{0}\right)$ is a disjoint union of klt pairs. Let $N_{i}=$ $\operatorname{ord}_{E_{i}}\left(g^{*} M\right)$, and let $v_{i}$ be the log discrepancy $a\left(E_{i}, X, \Delta\right)+1$ of $E_{i}$ with respect to $(X, \Delta)$. By our assumption,

$$
\left(N_{i}, v_{i}\right) \in \mathbb{Q}_{\geq 0} \times \mathbb{Q}_{\geq 0} \quad \text { for any } i .
$$

We assume that

$$
\left(N_{i}, v_{i}\right) \neq(0,0)
$$

for any divisor $E_{i}$. Denote by $r$ the least common multiple of the Cartier indices of $(X, \Delta)$ and $M$, that is, $r$ is the minimal positive integer such that both $r\left(K_{X}+\Delta\right)$ and $r M$ are Cartier.

Definition 1.1. We define the dlt motivic zeta function corresponding to the given data by

$$
\begin{aligned}
& Z_{\mathrm{mot}}^{\mathrm{dlt}}\left(X^{\mathrm{dlt}}, D^{\mathrm{dlt}}, M, K_{X}+\Delta, s\right) \\
& \quad:=\mathbb{L}^{-d} \sum_{J \subset I} \mathcal{E}_{\mathrm{st}}\left(E_{J}^{0}, D_{J}^{0}\right) \prod_{i \in J} \frac{(\mathbb{L}-1) \mathbb{L}^{-N_{i} s-v_{i}}}{1-\mathbb{L}^{-N_{i} s-v_{i}}} \in \mathcal{M}_{k}^{r}\left[\left[\mathbb{L}^{-s}\right]\right],
\end{aligned}
$$

where $\mathcal{M}_{k}^{r}$ is the finite extension of $\mathcal{M}_{k}$ obtained by adding $\mathbb{L}^{1 / r}$, and $\mathcal{E}_{\mathrm{st}}\left(E_{J}^{0}, D_{J}^{0}\right)$ is the stringy motive of the pair $\left(E_{J}^{0}, D_{J}^{0}\right)$ (see [Bat99; Vey03]).

Since we usually fix $M$ and $(X, \Delta)$ (but we may change $\left(X^{\mathrm{dlt}}, D^{\mathrm{dlt}}\right)$ ), we abuse notation and write this zeta function as $Z_{\text {mot }}^{\mathrm{dlt}}\left(X^{\mathrm{dlt}}, D^{\mathrm{dlt}}, s\right)$. It has the usual Euler number specialization

$$
Z_{\mathrm{top}}^{\mathrm{dlt}}\left(X^{\mathrm{dlt}}, D^{\mathrm{dlt}}, s\right):=\sum_{J \subset I} \chi_{\mathrm{st}}\left(E_{J}^{0}, D_{J}^{0}\right) \prod_{i \in J} \frac{1}{N_{i} s+v_{i}} \in \mathbb{Q}(s) .
$$

Our main theorem is the following. 
Theorem 1.2. For a fixed $M$ and $(X, \Delta)$ as before, if $\left(X_{1}^{\mathrm{dlt}}, D_{1}^{\mathrm{dlt}}\right)$ and $\left(X_{2}^{\mathrm{dlt}}, D_{2}^{\mathrm{dlt}}\right)$ are crepant birational equivalent dlt pairs (see (1.4)), we have

$$
Z_{\mathrm{mot}}^{\mathrm{dlt}}\left(X_{1}^{\mathrm{dlt}}, D_{1}^{\mathrm{dlt}}, s\right)=Z_{\mathrm{mot}}^{\mathrm{dlt}}\left(X_{2}^{\mathrm{dlt}}, D_{2}^{\mathrm{dlt}}, s\right) .
$$

In particular, the theorem says that $\left(N_{i}, v_{i}\right) \neq(0,0)$ for all $i \in I$ when we consider the components of $D_{1}^{\mathrm{dlt}}$ if and only if the same holds for the components of $D_{2}^{\mathrm{dlt}}$.

Suppose now that $f$ is a regular function on a smooth variety $X$. If we denote by $g:\left(X^{\mathrm{dlt}}, D^{\mathrm{dlt}}\right) \rightarrow X$ a dlt modification of $\left(X,(f=0)_{\text {red }}\right), \Delta=0$, $M=(f=0)$ and define the dlt motivic zeta function of $f$ by

$$
Z_{\mathrm{mot}}^{\mathrm{dlt}}(f, s):=Z_{\mathrm{mot}}^{\mathrm{dlt}}\left(X^{\mathrm{dlt}}, D^{\mathrm{dlt}}, M, K_{X}, s\right),
$$

then we get an immediate corollary.

Corollary 1.3. The dlt motivic zeta function $Z_{\mathrm{mot}}^{\mathrm{dlt}}(f, s)$ does not depend on the choice of dlt modification $\left(X^{\mathrm{dlt}}, D^{\mathrm{dlt}}\right)$.

We note that since different dlt modifications are crepant birationally equivalent to each other, they can be used to obtain useful information of singularities. For instance, it is proved in [ $\mathrm{dFKX} 12$ ] that dlt modifications can be used to define a finer topological invariant than the dual complex of a singularity. Our note is also motivated by this idea.

The paper is organized as follows. We prove Theorem 1.2 by studying the stratification provided by lc centers (see Definition 2.3) and compare two different ones in Section 3.1. This is inspired by the work in [dFKX12]. In Section 3.2, we point out a generalization of Batyrev's stringy Euler number for nonstrictly log canonical singularities. In Section 3.3, we also discuss an analogous definition in the case of degenerations of Calabi-Yau manifolds, for which the original global motivic zeta function is studied in [HN11; HN12]. Finally, in Section 4, we pose a few questions that could be interesting for further study.

Convention 1.4. We refer to [KM98] for the definitions of the basic notions used in birational geometry. Two pairs $\left(X_{i}, \Delta_{i}\right)(i=1,2)$ are called crepant birationally equivalent if there are birational proper morphisms $f_{i}: Y \rightarrow X_{i}$ such that

$$
f_{1}^{*}\left(K_{X_{1}}+\Delta_{1}\right)=f_{2}^{*}\left(K_{X_{2}}+\Delta_{2}\right)
$$

\section{Preliminaries}

In this section, we discuss some background.

\subsection{Motivic Zeta Function}

The motivic zeta function is the motivic upgrade of Igusa's $p$-adic zeta function. It was first introduced in [DL98] using the technique of motivic integration (see [DL99; DL98]). Here we briefly recall its definition, for more details, see [DL99, Paragraph 2.1]. 
We denote by $\mathcal{M}_{k}$ the ring $K_{0}\left(\operatorname{Var}_{k}\right)\left[\mathbb{L}^{-1}\right]$, where $K_{0}\left(\operatorname{Var}_{k}\right)$ is the Grothendieck ring of varieties over $k$, and $\mathbb{L}$ is the Lefschetz motive. For a smooth variety $X$ of pure dimension $d$ and a regular function $f: X \rightarrow \mathbb{A}^{1}$, let $\mathcal{L}_{n}(X)$ be the $n$ jet space of $X$, and $f_{n}$ the map on $n$-jet spaces induced by $f$. The motivic zeta function of $f$ is then defined as

$$
Z(s)=\sum_{n \geq 0}\left[X_{n}\right] \mathbb{L}^{-n d-n s} \in \mathcal{M}_{k}\left[\left[\mathbb{L}^{-s}\right]\right],
$$

where $X_{n}:=\left\{\gamma \in \mathcal{L}_{n}(X) \mid \operatorname{ord}_{t} f_{n}(\gamma)=n\right\}$ for $n \in \mathbb{Z}_{\geq 0}$.

As we mentioned in the introduction, an explicit formula for the motivic zeta function can be given in terms of a $\log$ resolution of $(X, D)$ as follows. Fix a $\log$ resolution $h: Y \rightarrow(X, D)$ that is an isomorphism over $X \backslash D$. Denote by $E_{i}$ $(i \in I)$ the irreducible components of the divisor $E=h^{-1}(D)$, and by $\left(N_{i}, v_{i}\right)$ the corresponding pair (mult $E_{i}\left(h^{*} D\right), a\left(E_{i}, X\right)+1$ ), where $a\left(E_{i}, X\right)$ is the discrepancy of $E_{i}$ with respect to $X$. For any subset $J$ of $I$, we put $E_{J}=\bigcap_{i \in J} E_{i}$ and $E_{J}^{0}=E_{J} \backslash \bigcup_{i \notin J} E_{i}$. We denote by $d$ the dimension of $X$. Then

$$
Z_{\text {mot }}(s)=\mathbb{L}^{-d} \sum_{J \subset I}\left[E_{J}^{0}\right] \prod \frac{(\mathbb{L}-1) \mathbb{L}^{-N_{i} s-v_{i}}}{1-\mathbb{L}^{-N_{i} s-v_{i}}} \in \mathcal{M}_{k}\left[\left[\mathbb{L}^{-s}\right]\right] .
$$

REMARK 2.1. Since the localized Grothendieck ring $\mathcal{M}_{k}$ is not a domain, we should specify what is meant by a pole of a rational function over $\mathcal{M}_{k}$. The definition we use is the following: if $Z\left(\mathbb{L}^{-s}\right)$ is an element of

$$
\mathcal{M}_{k}\left[\mathbb{L}^{-s}, \frac{1}{1-\mathbb{L}^{a-b s}}\right]_{(a, b) \in \mathbb{Z} \times \mathbb{Z}_{>0}} \subset \mathcal{M}_{k}\left[\left[\mathbb{L}^{-s}\right]\right],
$$

$s_{0}$ is a rational number, and $m$ is a nonnegative integer, then we say that $Z\left(\mathbb{L}^{-s}\right)$ has a pole at $s_{0}$ of order at most $m$ if there is a set $\mathcal{S}$ consisting of multisets in $\mathbb{Z} \times \mathbb{Z}_{>0}$ such that each element of $\mathcal{S}$ contains at most $m$ elements $(a, b)$ such that $a / b=s_{0}$ and $Z\left(\mathbb{L}^{-s}\right)$ belongs to the sub- $\mathcal{M}_{k}\left[\mathbb{L}^{-s}\right]$-module of $\mathcal{M}_{k}\left[\left[\mathbb{L}^{-s}\right]\right]$ generated by

$$
\left\{\frac{1}{\prod_{(a, b) \in S}\left(1-\mathbb{L}^{a-b s}\right)} \mid S \in \mathcal{S}\right\} .
$$

The same remark applies to $\mathcal{M}_{k}^{e}$.

Another application of motivic integration was to the definition of the stringy motive $\mathcal{E}_{\text {st }}(X, D)$ for a klt pair $(X, D)$ (see [Bat99; Vey03]).

Definition 2.2. For every klt pair $(X, D)$, let $Y \rightarrow(X, D)$ be a log resolution, and let $\left\{E_{i} \mid i \in I\right\}$ be the set consisting of exceptional divisors and birational transforms of the components of $D$. If for every $J \subset I$, we define $E_{J}^{0}$ as before, we then associate with $(X, D)$ the object

$$
\mathcal{E}_{\mathrm{st}}(X, D):=\sum_{J \subset I}\left[E_{J}^{0}\right] \prod_{i \in J} \frac{(1-\mathbb{L}) \mathbb{L}^{-a_{i}}}{\mathbb{L}^{-a_{i}}-1},
$$


where $0<a_{i}=a\left(E_{i}, X, D\right)+1$. This object lives in a finite extension of $\hat{\mathcal{M}}_{k}$ obtained by adding $\mathbb{L}^{1 / r}$, where $r$ is the Cartier index of $K_{X}+D$, and $\hat{\mathcal{M}}_{k}$ denotes Kontsevich's completed Grothendieck ring with respect to the decreasing filtration $\left(F_{m}\right)_{m \in \mathbb{N}}$ of $\mathcal{M}_{k}$, where $F_{m}$ is the subgroup of $M$ generated by the elements $[S] / L^{i}$ with $S$ an algebraic variety such that $\operatorname{dim}(S)-i \leq-m$. We call $\mathcal{E}_{\text {st }}(X, D)$ the stringy motive of $(X, D)$.

Obviously, if $\left(X_{i}, D_{i}\right)(i=1,2)$ are crepant birationally equivalent klt pairs, then we have

$$
\mathcal{E}_{\mathrm{st}}\left(X_{1}, D_{1}\right)=\mathcal{E}_{\mathrm{st}}\left(X_{2}, D_{2}\right)
$$

\subsection{Dlt Pairs}

For the reader's convenience, we give a short review of dlt singularities. For more background, see [KM98; Kol13].

Definition 2.3 (Dlt singularity and stratification). A pair $(X, D)$, with $X$ a normal variety $X$ and $D$ a $\mathbb{Q}$-divisor $D$ with coefficients belonging to [0,1], is called $d l t$ if $K_{X}+D$ is $\mathbb{Q}$-Cartier and if there is an open subset $U \subset X$ such that for any divisorial valuation $v$ with center contained in $X \backslash U$, we have the discrepancy $a(v, X, D)>-1$, and on $U$, all coefficients of $\left.D\right|_{U}$ are equal to 1 , and the pair $\left(U,\left.D\right|_{U}\right)$ has simple normal crossings.

Given a dlt pair $(X, D)$, let us write $\lfloor D\rfloor=E=\sum_{i \in I} E_{i}$. Then we know that for any $J \subset I$, the intersection $E_{J}=\bigcap_{i \in J} E_{i}$ is normal, and we call its components log canonical (lc) centers. If $W$ is a component of

$$
E_{J}^{0}=E_{J} \backslash \bigcup_{i \notin J} E_{i} \quad \text { and }\left.\quad\left(K_{X}+D\right)\right|_{W}=K_{W}+D_{W},
$$

then $\left(W, D_{W}\right)$ is a klt pair. Furthermore, if we denote by $\bar{W}$ the closure and write $\left.\left(K_{X}+D\right)\right|_{\bar{W}}=K_{\bar{W}}+D_{\bar{W}}$, then $\left(\bar{W}, D_{\bar{W}}\right)$ is dlt. We call the stratification of $X$ by components $W$ of $E_{J}^{0}$ the lc stratification. We call a component $W$ of $E_{J}^{0}$ an open stratum or simply a stratum.

We can define the dual complex $\mathcal{D} \mathcal{R}(X, D)=\mathcal{D} \mathcal{R}(E)$ as in [AFKX12, Def. 8]. This captures the combinatorial intersection information of closures of the strata.

Obviously, the notion of a dlt pair is a generalization of a simple normal crossing (snc) pair. In fact, it was first defined precisely to characterize the kind of singularities obtained after running MMP for an snc pair. The log canonical stratification is then a natural analogue of the snc stratification.

Definition 2.4 (Dlt modification). Let $X$ be a normal variety, and $D$ a $\mathbb{Q}$-divisor with coefficients in $[0,1]$. We say that $g^{\text {dlt }}: X^{\mathrm{dlt}} \rightarrow(X, D)$ is a dlt modification if the following holds: if $D^{\text {dlt }}$ is the sum of the divisorial part of the exceptional locus of $g^{\mathrm{dlt}}$ and of the birational transform of $D$, then $\left(X^{\mathrm{dlt}}, D^{\mathrm{dlt}}\right)$ is dlt, and $K_{X^{\text {dlt }}}+D^{\text {dlt }}$ is nef over $X$. 
For a pair $(X, D)$, a dlt modification can be constructed by running a relative MMP for a log resolution, where the boundary is chosen to be the sum of the exceptional divisor and of the birational transform $D$. When $K_{X}+D$ is $\mathbb{Q}$-Cartier, a dlt modification always exists by [OX12]. It is not unique in general, but we know that two dlt modifications $\left(X_{i}^{\mathrm{dlt}}, D_{i}^{\mathrm{dlt}}\right)(i=1,2)$ of $(X, D)$ are crepant birationally equivalent to each other, following the proof of [KM98, Theorem 3.52] (see also [dFKX12, Theorem 15]).

\section{Dlt Motivic Zeta Functions}

\subsection{Proof of Theorem 1.2}

Now we aim to prove Theorem 1.2, which says that the dlt motivic zeta function does not depend on the choice of dlt modification $X^{\mathrm{dlt}}$.

Proposition 3.1. Let $\left(X_{1}, D_{1}\right)$ and $\left(X_{2}, D_{2}\right)$ be two crepant birationally equivalent dlt pairs. If the birational map $X_{1} \rightarrow X_{2}$ gives an isomorphism between open subsets that contain all the generic points of the strata, then

$$
Z_{\mathrm{mot}}^{\mathrm{dlt}}\left(X_{1}, D_{1}, s\right)=Z_{\mathrm{mot}}^{\mathrm{dlt}}\left(X_{2}, D_{2}, s\right) .
$$

Proof. By our assumption, there is a one-to-one correspondence between the strata of $\left(X_{1}, D_{1}\right)$ and $\left(X_{2}, D_{2}\right)$. Since we know that $\left(X_{i}, D_{i}\right)$ are crepant birationally equivalent to each other, this implies that for each stratum $W_{1}$ that is a component of $\left(E_{J}^{0}\right)_{1}$, the correspondence gives a unique stratum $W_{2}$ such that if we write

$$
\left.\left(K_{X_{i}}+D_{i}\right)\right|_{W_{i}}=K_{W_{i}}+D_{W_{i}},
$$

then $\left(W_{i}, D_{W_{i}}\right)$ are crepant birationally equivalent. In particular, they have the same stringy motive. Therefore the corresponding summands in the expressions of $Z^{\mathrm{dlt}}\left(X_{i}, \Delta_{i}\right)$ are equal.

Proposition 3.2. Let $g: X_{1} \rightarrow X_{2}$ be a morphism of dlt modifications of a log canonical pair $(X, D)$ with an effective $\mathbb{Q}$-divisor $M$. Let $U \subset X_{2}$ be an open subset containing all lc centers of $\left(X_{2}, D_{2}\right)$ such that $\left(U,\left.D_{2}\right|_{U}\right)$ has simple normal crossings. If $\left(V=g^{-1}(U),\left.D_{1}\right|_{V}\right) \rightarrow\left(U,\left.D_{2}\right|_{U}\right)$ is the blow up of a stratum, then

$$
Z_{\mathrm{mot}}^{\mathrm{dlt}}\left(X_{1}, D_{1}, s\right)=Z_{\mathrm{mot}}^{\mathrm{dlt}}\left(X_{2}, D_{2}, s\right) \text {. }
$$

Proof. Let us assume that $V \rightarrow U$ is the blow up of $U$ along a connected component of $D_{J}^{U}=D_{J} \cap U$ for some $J$. Let $J^{\prime} \supset J$ and $G \subset D_{J^{\prime}}^{0}$ be an open stratum of $\left(X_{2}, D_{2}\right)$; thus,

$$
G^{U}:=G \cap U \subset D_{J^{\prime}}^{U}:=D_{J^{\prime}}^{0} \cap U \subset D_{J}^{U} .
$$

Let $W$ be the maximal stratum of $X_{1}$ that is over $G$. Over a Zariski neighborhood $G^{*}$ of the generic point of $G$,

$$
\bar{W} \cap g^{-1}\left(G^{*}\right)=G^{*} \times \mathbb{P}^{m}=\mathbb{P}_{G^{*}}^{m},
$$


where $m=\left|J^{\prime}\right|-1$. Furthermore, the birational transforms of the divisors $\left(D_{1}\right)_{j}$ $\left(i \in J^{\prime}\right)$ intersecting with $\bar{W} \cap g^{-1}\left(G^{*}\right)$ consist of $m+1$ coordinate hyperplane sections $\left(x_{j}=0\right)$ on $\mathbb{P}_{G^{*}}^{m}$.

Write $\left.\left(K_{X_{2}}+D_{2}\right)\right|_{\bar{W}}=K_{\bar{W}}+D_{\bar{W}}$ and $\left.\left(K_{X_{1}}+D_{1}\right)\right|_{G}=K_{G}+D_{G}$. We claim that $\left(\bar{W}, D_{\bar{W}}\right)$ is crepant birationally equivalent to

$$
\left(G, D_{G}\right) \times\left(\mathbb{P}^{m}, T:=\sum_{i=1}^{m}\left(x_{i}=0\right)\right):=\left(G \times \mathbb{P}^{m}, D_{G} \times \mathbb{P}^{m}+G \times T\right) .
$$

In fact, if we take $p: W^{\prime} \rightarrow \bar{W}$ and $q: W^{\prime} \rightarrow G \times \mathbb{P}^{m}$ to be a common resolution, then we know that

$$
p^{*}\left(K_{\bar{W}}+D_{\bar{W}}\right)-q^{*}\left(K_{G \times \mathbb{P}^{m}}+G \times T\right)
$$

is a vertical divisor, and hence it is the pull back of $D_{G}$.

In order to compute $Z_{\mathrm{mot}}^{\mathrm{dlt}}\left(X_{2}, D_{2}, s\right)-Z_{\text {mot }}^{\mathrm{dlt}}\left(X_{1}, D_{1}, s\right)$, since the product of a $\log$ resolution of $\left(G, D_{G}\right)$ with $\left(\mathbb{P}^{m}, T\right)$ also gives a $\log$ resolution of $\left(G, D_{G}\right) \times$ $\left(\mathbb{P}^{m}, T\right)$, we can compare the difference on each piece and hence reduce to the case where $G$ is a point.

Write $J^{\prime}=J \cup J_{1}$, where $J$ and $J_{1}$ are disjoint. In $Z_{\text {mot }}^{\text {dlt }}\left(X_{1}, D_{1}, s\right)$, the contribution is

$$
\prod_{j \in J} \frac{(\mathbb{L}-1) \mathbb{L}^{-N_{i} s-v_{i}}}{1-\mathbb{L}^{-N_{i} s-v_{i}}} \cdot \prod_{j \in J_{1}} \frac{(\mathbb{L}-1) \mathbb{L}^{-N_{i} s-v_{i}}}{1-\mathbb{L}^{-N_{i} s-v_{i}}} .
$$

If $E_{0}$ is the exceptional divisor of $X_{2} \rightarrow X_{1}$, then its multiplicity along $M$ is $N_{0}=$ $\sum_{i \in J} N_{i}$, and by the $\log$ pull-back formula we have $v_{0}=\sum_{i \in J} v_{i}$. Therefore, in $Z_{\text {mot }}^{\text {dlt }}\left(X_{2}, D_{2}, s\right)$, the contribution is

$$
\begin{aligned}
\sum_{K \subset J, K \neq J}(\mathbb{L}-1)^{|J|-|K|-1} \frac{(\mathbb{L}-1) \mathbb{L}^{-N_{0} s-v_{0}}}{1-\mathbb{L}^{-N_{0} s-v_{0}}} \cdot \prod_{j \in K} \frac{(\mathbb{L}-1) \mathbb{L}^{-N_{i} s-v_{i}}}{1-\mathbb{L}^{-N_{i} s-v_{i}}} \\
\cdot \prod_{j \in J_{1}} \frac{(\mathbb{L}-1) \mathbb{L}^{-N_{i} s-v_{i}}}{1-\mathbb{L}^{-N_{i} s-v_{i}}} .
\end{aligned}
$$

The fact that the two contributions are the same follows from the simple equality

$$
\prod_{i \in J} \frac{1}{t_{i}-1}=\frac{1}{\prod_{i \in J} t_{i}-1} \cdot \sum_{K \subset J, K \neq J} \prod_{i \in K} \frac{1}{t_{i}-1} .
$$

Now we can prove Theorem 1.2.

Proof of (1.2). We first take a $\log$ resolution $\tilde{X}_{j}$ of $\left(X_{j}^{\mathrm{dlt}}, D_{j}^{\mathrm{dlt}}\right)(j=1$ or 2$)$. Furthermore, by the weak factorization theorem (see [AKMW02]) we can connect $\tilde{X}_{1}$ and $\tilde{X}_{2}$ by

$$
\tilde{X}_{1}=Y_{1-\rightarrow \cdots-\rightarrow Y_{m}=\tilde{X}_{2}}
$$

such that

(1) each $\left(Y_{i}, E_{Y_{i}}\right)$ admits a morphism to either $\tilde{X}_{1}$ or $\tilde{X}_{2}$; 
(2) if $Y_{i}$ admits a morphism to $\tilde{X}_{j}(j=1$ or 2$)$, then $\left(Y_{i}, E_{Y_{i}}\right)$ is a $\log$ resolution of ( $\left.X_{j}^{\mathrm{dlt}}, D_{j}^{\mathrm{dlt}}\right)$, where $E_{Y_{i}}$ denotes the sum of the reduced exceptional divisor over $X_{j}^{\mathrm{dlt}}$ and of the birational transform of $D_{j}^{\mathrm{dlt}}$, and

(3) $Y_{i} \rightarrow Y_{i+1}$ is either a blow up of a smooth irreducible center $G_{i}$ having simple normal crossing with $E_{Y_{i}}$ or the inverse of such a blow up.

Assume that $\psi_{i}: Y_{i+1} \rightarrow Y_{i}$ is a blow up; otherwise, we just reverse the arrow.

Running a relative MMP of $K_{Y_{i}}+E_{Y_{i}}$ over $X_{j}^{\mathrm{dlt}}$ following [BCHM10], we obtain a dlt modification $\left(Y_{i}^{\mathrm{dlt}}, \Delta_{i}^{\mathrm{dlt}}\right) \rightarrow\left(X_{j}^{\mathrm{dlt}}, D_{j}^{\mathrm{dlt}}\right)$. If $Y_{i} \rightarrow Y_{i}^{\mathrm{dlt}}$ is not an isomorphism around $G_{i}$, then we know that for the exceptional divisor $v_{i}$ of $\psi_{i}$, we have

$$
a\left(v_{i}, Y_{i}^{\mathrm{dlt}}, \Delta_{i}^{\mathrm{dlt}}\right)>-1 .
$$

Thus, if we run MMP of $\left(Y_{i+1}, E_{Y_{i+1}}\right)$ over $X_{j}^{\mathrm{dlt}}$, we obtain a dlt modification $Y_{i+1}^{\mathrm{dlt}}$ that is isomorphic to $Y_{i}^{\mathrm{dlt}}$ near the generic points of all the lc centers. Therefore, we can apply Proposition 3.1 to conclude that they give the same dlt motivic zeta function, that is,

$$
Z_{\mathrm{mot}}^{\mathrm{dlt}}\left(Y_{i+1}^{\mathrm{dlt}}, \Delta_{i+1}^{\mathrm{dlt}}, s\right)=Z_{\mathrm{mot}}^{\mathrm{dlt}}\left(Y_{i}^{\mathrm{dlt}}, \Delta_{i}^{\mathrm{dlt}}, s\right) .
$$

If $Y_{i} \rightarrow Y_{i}^{\text {dlt }}$ is an isomorphism around $G_{i}$, then we can find an open subset $U_{i} \subset Y_{i}^{\mathrm{dlt}}$ such that if we take the blow up of $\left.G_{i}\right|_{U_{i}}$, then we get an open subset of $Y_{i+1}^{\mathrm{dlt}}$ that also meets all the lc centers of $\left(Y_{i+1}^{\mathrm{dlt}}, \Delta_{i+1}^{\mathrm{dlt}}\right)$. By [dFKX12, Lemma 36] we can extend $U_{i+1} \rightarrow U_{i}$ to a dlt modification $\left(\left(Y_{i+1}^{\prime}\right)^{\mathrm{dlt}},\left(\Delta_{i+1}^{\prime}\right)^{\mathrm{dlt}}\right)$ of $\left(Y_{i}^{\mathrm{dlt}}, \Delta_{i}^{\mathrm{dlt}}\right)$. Thus, we apply Proposition 3.2 for

$$
\left(\left(Y_{i+1}^{\prime}\right)^{\mathrm{dlt}},\left(\Delta_{i+1}^{\prime}\right)^{\mathrm{dlt}}\right) \rightarrow\left(Y_{i}^{\mathrm{dlt}}, \Delta_{i}^{\mathrm{dlt}}\right) \rightarrow\left(X_{j}^{\mathrm{dlt}}, D_{j}^{\mathrm{dlt}}\right)
$$

and conclude that

$$
Z_{\mathrm{mot}}^{\mathrm{dlt}}\left(Y_{i}^{\mathrm{dlt}}, \Delta_{i}^{\mathrm{dlt}}, s\right)=Z_{\mathrm{mot}}^{\mathrm{dlt}}\left(\left(Y_{i+1}^{\prime}\right)^{\mathrm{dlt}},\left(\Delta_{i+1}^{\prime}\right)^{\mathrm{dlt}}, s\right) .
$$

Since $Y_{i+1}^{\mathrm{dlt}} \rightarrow\left(Y_{i+1}^{\prime}\right)^{\mathrm{dlt}}$ is an isomorphism near the generic points of all the lc centers, we again know that

$$
Z_{\mathrm{mot}}^{\mathrm{dlt}}\left(Y_{i+1}^{\mathrm{dlt}}, \Delta_{i+1}^{\mathrm{dlt}}, s\right)=Z_{\mathrm{mot}}^{\mathrm{dlt}}\left(\left(Y_{i+1}^{\prime}\right)^{\mathrm{dlt}},\left(\Delta_{i+1}^{\prime}\right)^{\mathrm{dlt}}, s\right) .
$$

\subsection{A Generalization of Batyrev's Stringy Invariant}

Let $(X, D)$ be a log pair such that $K_{X}+D$ is $\mathbb{Q}$-Cartier. We assume that $(X, D)$ does not have strictly lc singularities. Let $g^{\mathrm{dlt}}:\left(X^{\mathrm{dlt}}, D^{\mathrm{dlt}}\right) \rightarrow(X, D)$ be a dlt modification. We write

$$
K_{X^{\mathrm{dlt}}}+D^{\mathrm{dlt}}+M=\left(g^{\mathrm{dlt}}\right)^{*}\left(K_{X}+D\right)
$$

and $M=\sum_{i} N_{i} E_{i}$, where the sum is over all the exceptional divisors $E_{i}$ of $g^{\mathrm{dlt}}$. Then by our assumption that $(X, D)$ does not have strictly log canonical singularities we know that the coefficient $N_{i}$ of $M$ along a $g^{\text {dlt }}$-exceptional component is always positive. 
Definition-Proposition 3.3. We define the stringy motive for a $d$-dimensional $\log$ pair $(X, D)$ without strictly log canonical singularities to be

$$
\begin{aligned}
\mathcal{E}_{\mathrm{st}}(X, D) & =\mathbb{L}^{d} \cdot Z_{\mathrm{mot}}^{\mathrm{dlt}}\left(X^{\mathrm{dlt}}, D^{\mathrm{dlt}}, M, K_{X^{\mathrm{dlt}}}+D^{\mathrm{dlt}}, 1\right) \\
& =\sum_{J \subset I} \mathcal{E}_{\mathrm{st}}\left(E_{J}^{0}, D_{J}^{0}\right) \prod_{i \in J} \frac{(\mathbb{L}-1) \mathbb{L}^{-N_{i}}}{1-\mathbb{L}^{-N_{i}}},
\end{aligned}
$$

which lives in a finite extension $\hat{\mathcal{M}}_{k}^{r}$ of $\hat{\mathcal{M}}_{k}$ obtained by adding $\mathbb{L}^{1 / r}$, where $r$ is the least common multiple of the Cartier index of $\left(X^{\mathrm{dlt}}, D^{\mathrm{dlt}}\right)$ and $N_{i}$. Similarly, we can define its Euler number

$$
\chi_{\mathrm{st}}(X, D)=\sum_{J \subset I} e_{\mathrm{st}}\left(E_{J}^{0}, D_{J}^{0}\right) \prod_{i \in J} \frac{1}{N_{i}},
$$

where $e_{\mathrm{st}}\left(E_{J}^{0}, D_{J}^{0}\right)$ is Batyrev's stringy Euler number of a klt pair (see [Bat99; Vey03]).

If $\left(X_{i}^{\mathrm{dlt}}, D_{i}^{\mathrm{dlt}}\right) \rightarrow(X, D)(i=1,2)$ are two dlt modifications of $(X, D)$ and $p_{i}: Y \rightarrow\left(X_{i}^{\mathrm{dlt}}, D_{i}^{\mathrm{dlt}}\right)$ is a common resolution, then

$$
p_{1}^{*}\left(K_{X_{1}^{\mathrm{dlt}}}+D_{1}^{\mathrm{dlt}}\right)=p_{2}^{*}\left(K_{X_{2}^{\mathrm{dlt}}}+D_{2}^{\mathrm{dlt}}\right) \quad \text { and } \quad p_{1}^{*} M_{1}=p_{2}^{*} M_{2} \text {. }
$$

These are the main properties that were used in the proof of Theorem 1.2. More precisely, by [dFKX12, Proposition 37] we know that we can find dlt modifications $g_{i}:\left(\tilde{X}_{i}^{\mathrm{dlt}}, \tilde{D}_{i}^{\mathrm{dlt}}\right) \rightarrow\left(X_{i}^{\mathrm{dlt}}, D_{i}^{\mathrm{dlt}}\right)$ such that $\tilde{X}_{1}$ and $\tilde{X}_{2}$ are isomorphic around the generic points of all $\log$ canonical centers of $\left(\tilde{X}_{1}^{\mathrm{dlt}}, \tilde{D}_{1}^{\mathrm{dlt}}\right)$ and $\left(\tilde{X}_{2}^{\mathrm{dlt}}, \tilde{D}_{2}^{\mathrm{dlt}}\right)$. We then have

$$
\begin{aligned}
Z_{\mathrm{mot}}^{\mathrm{dlt}} & \left(X_{1}^{\mathrm{dlt}}, D_{1}^{\mathrm{dlt}}, M_{1}, K_{X_{1}^{\mathrm{dlt}}}+D_{1}^{\mathrm{dlt}}, 1\right) \\
& =Z_{\mathrm{mot}}^{\mathrm{dlt}}\left(\tilde{X}_{1}^{\mathrm{dlt}}, \tilde{D}_{1}^{\mathrm{dlt}}, g_{1}^{*} M_{1}, K_{X_{1}^{\mathrm{dlt}}}+D_{1}^{\mathrm{dlt}}, 1\right) \quad \text { (by Theorem 1.2) } \\
& =Z_{\mathrm{mot}}^{\mathrm{dlt}}\left(\tilde{X}_{2}^{\mathrm{dlt}}, \tilde{D}_{2}^{\mathrm{dlt}}, g_{2}^{*} M_{2}, K_{X_{2}^{\mathrm{dlt}}}+D_{2}^{\mathrm{dlt}}, 1\right) \quad(\text { by Proposition 3.1) } \\
& =Z_{\mathrm{mot}}^{\mathrm{dlt}}\left(X_{2}^{\mathrm{dlt}}, D_{2}^{\mathrm{dlt}}, M_{2}, K_{X_{2}^{\mathrm{dlt}}}+D_{2}^{\mathrm{dlt}}, 1\right) \quad \text { (by Theorem 1.2). }
\end{aligned}
$$

Therefore, we conclude that the previous definition of stringy motive does not depend on the choice of a dlt modification.

When $(X, D)$ is $\log$ terminal, then $X^{\mathrm{dlt}}=X$, and $\mathcal{E}_{\mathrm{st}}(X, D)$ is trivially equal to Batyrev's original definition in [Bat99].

REMARK 3.4. In [Vey03, Formula 3.2.1], Veys also attempted to give a definition

$$
\mathcal{Z}^{\mathrm{V}}(s) \in \mathcal{M}_{k}^{r}\left[\left[L^{-s}\right]\right],
$$

which aims to generalize Batyrev's stringy motive by taking $s=1$. The most general category of singularities for which Veys can possibly define a stringy invariant is also nonstrictly lc singularities. However, in Section 5 of [Vey03], a technical difficulty is discussed. This comes up since there can be log discrepancy 0 valuations even if the singularity is not strictly lc. However, this difficulty does not appear in our definition since we only consider dlt modifications. 
In general, our definition is different from $\mathcal{Z}^{\mathrm{V}}(1)$ since if we calculate everything on a $\log$ resolution, then it is clear that $\mathcal{Z}^{\mathrm{V}}(1)$ takes a sum over more strata than ours. The relationship between the two extensions is worthy of further investigation.

\subsection{Global Setting for Degenerations of Calabi-Yau Varieties}

In [HN11; HN12], a global version of motivic zeta function is introduced for a degeneration of a smooth Calabi-Yau variety. A similar question to the monodromy conjecture is then asked in this setting. We can introduce a dlt motivic zeta function in this global case, whose only pole is equal to the minimal weight.

We will use the following formula as the definition of the motivic zeta function. For the original conceptual definition, we refer to [HN11; HN12].

Definition 3.5. Fix $R$ to be a complete DVR. Let $X / K$ be a Calabi-Yau projective smooth variety, that is, $K_{X}$ is trivial. Let $\mathfrak{X}$ be an sncd projective model of $X$ over $\operatorname{Spec}(R)$, that is, $\mathfrak{X} \times \operatorname{Spec}(R) \operatorname{Spec}(K)=X$, and $\left(\mathfrak{X},\left(\mathfrak{X}_{0}\right)_{\text {red }}\right)$ is snc, where $\left(\mathfrak{X}_{0}\right)_{\text {red }}=\sum_{i \in I} E_{i}$ is the special fiber. We fix a canonical form $\omega$ on $\mathfrak{X}$ whose restriction on $X$ gives the volume form of $X$. Write $\mathfrak{X}_{0}=\sum_{i \in I} N_{i} E_{i}$ and let $\mu_{i}-1$ be the vanishing order of $\omega$ along $E_{i}$. We call $\mu_{i} / N_{i}$ the weight of $E_{i}$. Then the minimal weight is $\min _{i}\left\{\mu_{i} / N_{i}\right\}$, which, as we easily see, does not depend on the choice of the model $\mathfrak{X}$.

For any $J \subset I$, let $E_{J}=\bigcap_{i \in J} E_{i}$, and denote by $\pi$ a uniformizing parameter of $R$. Let $N_{J}=\operatorname{gcd}\left\{N_{i} \mid i \in J\right\}$, and let $\mathfrak{Y}$ be the normalization of

$$
\mathfrak{X} \times \operatorname{Spec}(R) \operatorname{Spec}(R[x]) /\left(x^{N_{J}}-\pi\right) .
$$

Then we denote by $\tilde{E}_{J}^{0}$ the preimage of $E_{J}^{0}$ under the morphism $\mathfrak{Y} \rightarrow \mathfrak{X}$. We know that $\tilde{E}_{J}^{0} \rightarrow E_{J}^{0}$ is an étale morphism (see [DL02, Paragraph 2.3], [NS07, Sect. 4]).

With this notation, the motivic zeta function of $(X, \omega)$ is

$$
Z_{X, \omega}(s)=\sum_{\emptyset \neq J \subset I}(\mathbb{L}-1)^{|J|-1}\left[\tilde{E}_{J}^{0}\right] \prod_{i \in J} \frac{\mathbb{L}^{-s N_{i}-\mu_{j}}}{1-\mathbb{L}^{-s N_{i}-\mu_{i}}} .
$$

We can similarly define the dlt motivic zeta function. A model $\mathfrak{X}^{\text {dlt }}$ of $X / K$ is a minimal dlt model if $\left(\mathfrak{X}^{\text {dlt }},\left(\mathfrak{X}_{0}^{\text {dlt }}\right)_{\text {red }}\right)$ is dlt and $K_{\mathfrak{X}^{\text {dlt }}}+\left(\mathfrak{X}_{0}^{\text {dlt }}\right)_{\text {red }} \sim_{\mathbb{Q}} 0$. We note that since $K_{X} \sim 0$, semi-upper-continuity implies that in fact we have

$$
K_{\mathfrak{X}^{\mathrm{dlt}}}+\left(\mathfrak{X}_{0}^{\mathrm{dlt}}\right)_{\text {red }} \sim 0 .
$$

It follows from the existence of resolutions of singularities and MMP (see [BCHM10; HX13]) that such minimal dlt models exist; in fact, a minimal dlt model can be obtained by running MMP on $\left(\mathfrak{X},\left(\mathfrak{X}_{0}\right)_{\text {red }}\right)$ from an sncd model $\mathfrak{X}$ of $X / K$. Given a minimal dlt model $\mathfrak{X}^{\text {dlt }}$, write

$$
\left(\mathfrak{X}_{0}^{\mathrm{dlt}}\right)_{\text {red }}=\sum_{i \in I_{0}} E_{i} \quad \text { and } \quad \mathfrak{X}_{0}^{\mathrm{dlt}}=\sum_{i \in I_{0}} N_{i} E_{i} .
$$


For any $J \subset I_{0}$, let $E_{J}=\bigcap_{i \in J} E_{j}$ and $E_{J}^{0}=E_{J} \backslash \bigcup_{i \notin J} E_{i}$. Let $\tilde{E}_{J}^{0}$ be the preimage of $E_{J}^{0}$ by the morphism $\mathfrak{Y} \rightarrow \mathfrak{X}^{\text {dlt }}$, where $\mathfrak{Y}$ is the normalization of

$$
\mathfrak{X}^{\text {dlt }} \times \operatorname{Spec}(R) \operatorname{Spec}(R[x]) /\left(x^{N_{J}}-\pi\right),
$$

and $N_{J}=\operatorname{gcd}\left\{N_{i} \mid i \in J\right\}$. We then write

$$
\left.\left(K_{\mathfrak{X}^{\mathrm{dlt}}}+\left(\mathfrak{X}_{0}^{\mathrm{dlt}}\right)_{\mathrm{red}}\right)\right|_{\tilde{E}_{J}^{0}}=\left.\left(K_{\mathfrak{Y}}+\left(\mathfrak{Y}_{0}\right)_{\mathrm{red}}\right)\right|_{\tilde{E}_{J}^{0}}=K_{\tilde{E}_{J}^{0}}+\tilde{D}_{J}^{0} .
$$

Since $E_{J}^{0}$ is an open stratum, we know that

$$
\left.\left(K_{\mathfrak{X}^{\mathrm{dlt}}}+\left(\mathfrak{X}_{0}^{\mathrm{dlt}}\right)_{\mathrm{red}}\right)\right|_{E_{J}^{0}}=K_{E_{J}^{0}}+D_{J}^{0}
$$

is a klt pair. Since the pull back of $K_{E_{J}^{0}}+D_{J}^{0}$ on $\tilde{E}_{J}^{0}$ is $K_{\tilde{E}_{J}^{0}}+\tilde{D}_{J}^{0}$, this implies that $\left(\tilde{E}_{J}^{0}, \tilde{D}_{J}^{0}\right)$ is klt. Therefore, we can make the following definition.

Definition 3.6. The dlt motivic zeta function of $(X, \omega)$ is

$$
Z_{X, \omega}^{\mathrm{dlt}}(s)=\sum_{\emptyset \neq J \subset I_{0}}(\mathbb{L}-1)^{|J|-1} \mathcal{E}_{\mathrm{st}}\left(\tilde{E}_{J}^{0}, \tilde{D}_{J}^{0}\right) \prod_{i \in J} \frac{\mathbb{L}^{-s N_{i}-\mu_{i}}}{1-\mathbb{L}^{-s N_{i}-\mu_{i}}},
$$

where $\mu_{i}$ is the vanishing order of $\omega$ on $E_{i}$.

Although two minimal dlt models are crepant birational to each other, we cannot directly apply Theorem 1.2 because in the definition we use $\tilde{E}_{J}^{0}$ instead of $E_{J}^{0}$ itself. However, we can apply the argument in the proof of Theorem 1.2.

More precisely, let us assume that $\mathfrak{X}_{2}^{\text {dlt }} \rightarrow \mathfrak{X}_{1}^{\text {dlt }}$ is obtained by compactifying the blow up of a stratum $E_{J}^{0}$ as in Proposition 3.2. Then for a fixed proper subset $K$ of $J$ and another subset $J_{1} \subset I$ that is disjoint with $J$, we consider the corresponding stratum $\tilde{F}_{K \cup J_{1}}^{0}$ on $\mathfrak{X}_{2}^{\text {dlt }}$, which is over $\tilde{E}_{J \cup J_{1}}^{0}$ on $\mathfrak{X}_{1}^{\text {dlt }}$ with the fiber being an étale cover of $\mathbb{G}_{m}^{|J|-|K|-1}$ described in [NS07, Lemma 7.4], where we have $a=1$. Therefore, $\tilde{F}_{K \cup J_{1}}^{0}$ is also a $\mathbb{G}_{m}^{|J|-|K|-1}$-bundle over $\tilde{E}_{J \cup J_{1}}^{0}$ by [NS07, 7.4]. Thus, the previous calculation in Proposition 3.2 still holds.

TheOrem 3.7. $Z_{X, \omega}^{\mathrm{dlt}}(s)$ does not depend on the choice of minimal dlt models.

REMARK 3.8. Since $\mu_{j}-1$ is the vanishing order of $\omega$ along $E_{i}$ and

$$
K_{\mathfrak{X}^{\mathrm{dlt}}}+\left(\mathfrak{X}_{0}^{\mathrm{dlt}}\right)_{\text {red }} \sim 0,
$$

we know that for every $i \in I_{0}, v_{i} / N_{i}$ is equal to the minimal value among $\left\{v_{j} / N_{j} \mid\right.$ $j \in I\}$.

\section{Questions}

The discussion in the previous sections leaves open a few questions.

The first conceptually important one is to give an intrinsic construction of $Z_{\text {mot }}^{\text {dlt }}(f, s)$. 
Question 4.1. Find a motivic integral definition of $Z_{\text {mot }}^{\text {dlt }}(f, s)$ without passing to the dlt modification.

Secondly, it is natural to consider the poles of $Z_{\text {mot }}^{\text {dlt }}(f, s)$. In particular, we can ask similar questions to the monodromy conjecture for $Z_{\text {mot }}^{\text {dlt }}(f, s)$ :

Question 4.2. For a pole $s$ of $Z_{\text {mot }}^{\mathrm{dlt}}(f, s)$, we conjecture that the following is true:

(weak) $e^{2 \pi i s}$ is an eigenvalue of the local monodromy action on the Milnor fiber of $f$ at some point $x \in(f=0)$,

(strong) $s$ is a root of Berstein-Sato polynomial.

In general, the sets of poles of $Z_{\mathrm{mot}}$ and $Z_{\mathrm{mot}}^{\mathrm{dlt}}$ could be different, as illustrated by the following simple example.

EXAMPLE 4.3. Consider a general degree $d>1$ homogenous equation $f_{d}\left(x_{1}, \ldots\right.$, $\left.x_{n}\right)=0$ and consider its zero locus $D$ in $\mathbb{A}^{n}$, which has a cone singularity at the origin.

When $d<n,\left(\mathbb{A}^{n}, D\right)$ is plt, and the only pole of $Z_{\text {mot }}^{\mathrm{dlt}}$ is -1 . To calculate the poles of $Z_{\text {mot }}(s)$, we take the log resolution by blowing up the origin. Then we see that there is another pole $-\frac{n}{d}$ for $Z_{\text {mot }}(s)$.

When $d \geq n$, the dlt modification and the log resolution are given by blowing up the original point, and so for both functions, the poles are -1 and $-\frac{n}{d}$.

In fact, if we denote a dlt modification by

$$
g^{\mathrm{dlt}}:\left(X^{\mathrm{dlt}}, D^{\mathrm{dlt}}\right) \rightarrow\left(X, D_{\mathrm{red}}\right),
$$

where $D=(f=0)$, then by the definition of the dlt modification we have

$$
\left(g^{\mathrm{dlt}}\right)^{*}\left(K_{X}+D\right) \geq K_{X^{\mathrm{dlt}}}+D^{\mathrm{dlt}} .
$$

Therefore, all pole candidates for $Z_{\text {mot }}^{\text {dlt }}(s)$ lie in the interval $[-1,0]$. A more precise question concerning the relation between the two zeta functions can be asked as follows.

Question 4.4. Are the poles of the dlt motivic zeta function $Z_{\text {mot }}^{\mathrm{dlt}}(f, s)$ always poles of $Z_{\text {mot }}(f, s)$ ?

We admit that we do not have much evidence for these questions.

We further provide an example of a four-variable function whose (regular) motivic zeta function has a pole in $[-1,0]$ that is not a pole of the dlt motivic zeta function.

EXAMPLE 4.5. We consider the polynomial

$$
f=u^{N-2}\left(v^{2}+w^{2}+x^{2}\right)+v^{N}+w^{N}+x^{N}+u^{N+2} \in k[u, v, w, x] .
$$

We denote by $D$ the zero locus of $f$ in $X=\mathbb{A}^{4}$, which has an isolated singularity at the origin. Let $Y_{1} \rightarrow X$ be the blow up at the origin. We denote the exceptional 
divisor by $E_{1} \cong \mathbb{P}^{3}$. The strict transform $D_{1}$ of $D$ on $Y_{1}$ has a unique singular point $y_{1}$ that is an $A_{1}$-singularity: on the blow-up chart with coordinates

$$
\left(u, v^{\prime}=v / u, w^{\prime}=w / u, x^{\prime}=x / u\right),
$$

the divisor $D_{1}$ is defined by the equation

$$
x^{\prime 2}+v^{\prime 2}+w^{\prime 2}+\left(x^{\prime}\right)^{N}+\left(v^{\prime}\right)^{N}+\left(w^{\prime}\right)^{N}+u^{2}=0,
$$

and $y_{1}$ is the point $(0,0,0)$.

We claim that if $N \geq 4$, then $\left(Y_{1}, D_{1}+E_{1}\right)$ is a dlt modification of $\left(\mathbb{A}^{3}, D\right)$. In fact, we know that

$$
\left.\left(K_{Y_{1}}+D_{1}+E_{1}\right)\right|_{E_{1}}=K_{\mathbb{P}^{3}}+D^{\prime}
$$

is nef, where $\operatorname{deg}\left(D^{\prime}\right)=N$, and $\left(Y_{1}, D_{1}+E_{1}\right)$ is dlt.

Let $Y_{2} \rightarrow Y_{1}$ be the blow up at $y_{1}$ with the exceptional divisor $E_{2}$, and denote by $D_{2}$ and $E_{1}^{\prime}$ the strict transforms of $D_{1}$ and $E_{1}$ on $Y_{2}$, respectively. The composed morphism $h: Y_{2} \rightarrow X$ is a log resolution for the pair $(X, D)$. We have

$$
h^{*} D=D_{2}+N E_{1}^{\prime}+(N+2) E_{2} \text { and } K_{Y_{2} / \mathbb{A}^{3}}=3 E_{1}^{\prime}+6 E_{2} .
$$

Thus, the possible poles along of $D$ at $D_{2}, E_{1}^{\prime}$, and $E_{2}$ are given by $1,4 / N$, and $7 /(N+2)$, respectively.

Clearly, $E_{2} \cong \mathbb{P}^{3}$, and $E_{1}^{\prime}$ is isomorphic to the blow up of a point on $\mathbb{P}^{3}$. The intersection of the divisors $D_{1}$ and $E_{1}$ on $Y_{1}$ is a surface $S$, which is defined by the equation

$$
u=x^{\prime 2}+v^{\prime 2}+w^{\prime 2}+\left(x^{\prime}\right)^{N}+\left(v^{\prime}\right)^{N}+\left(w^{\prime}\right)^{N}=0
$$

on the blow-up chart with coordinates $\left(u, v^{\prime}, w^{\prime}, x^{\prime}\right)$ as before. The surface $S$ has precisely one singularity at $y_{1}$, which is of $A_{1}$-type, so that $D_{2} \cap E_{1}^{\prime} \cap E_{2}$ consists of a conic curve, and

$$
E_{1}^{\prime} \cap E_{2} \cong \mathbb{P}^{2} \quad \text { and } \quad E_{2} \cap D_{2} \cong \mathbb{P}^{1} \times \mathbb{P}^{1}
$$

To make the result more transparent, we compute the topological specializations $Z_{\text {top }}(f ; s)$ and $Z_{\text {top }}^{\text {dlt }}(f ; s)$ using the models $Y_{2}$ and $Y_{1}$, respectively. We first obtain

$$
\begin{aligned}
Z_{\text {top }}(f, s)= & \chi\left(\mathbb{A}^{3} \backslash D\right)+\frac{\chi(D)-1}{s+1}+\frac{5-N^{3}+4 N^{2}-6 N}{N s+4}+\frac{-1}{(N+2) s+7} \\
& +\frac{N^{3}-4 N^{2}+6 N-2}{(s+1)(N s+4)}+\frac{2}{(s+1)((N+2) s+7)} \\
& +\frac{1}{(N s+4)((N+2) s+7)}+\frac{2}{(s+1)((N+2) s+7)(N s+4)},
\end{aligned}
$$

where we use the fact that $\chi(S)=N^{3}-4 N^{2}+6 N-1$. Therefore, if $N>5$, then the (regular) zeta function has three distinct poles $-\frac{4}{N},-\frac{7}{N+2}$, and -1 contained in $[-1,0]$. On the other hand, since $E_{1}$ is the unique exceptional divisor of $Y_{1} / \mathbb{A}^{3}$, the dlt zeta function $Z_{\text {top }}^{\text {dlt }}(f, s)$ only has poles at $\frac{4}{N}$ and -1 . 
We also note that in the global setting for a degeneration of Calabi-Yau varieties, the only pole of $Z_{\mathrm{mot}}^{\mathrm{dlt}}(X, s)$ is the minimal weight $s$. It was shown in [HN12, Theorem 6.7] that $s$ is a pole of $Z_{\text {mot }}(X, s)$, and $e^{-2 \pi i s}$ is an eigenvalue of the monodromy action on $H^{d}\left(X_{\bar{K}}, \mathbb{Q}_{l}\right)$ where $d=\operatorname{dim}(X)$.

AcKnowledgments. We would like to thank Stefan Kebekus, Johannes Nicaise, Vim Veys, and Ziquan Zhuang for inspiring discussions. We also want to thank the referee for many helpful suggestions on the exposition. A large part of this work was done while the author visited FRIAS as a "Senior External Scholar". We would like to thank Stefan Kebekus for invitation and for the wonderful environment provided there.

\section{References}

[AKMW02] D. Abramovich, K. Karu, K. Matsuki, and J. Włodarczyk, Torification and factorization of birational maps, J. Amer. Math. Soc. 15 (2002), no. 3, 531572.

[Bat99] V. Batyrev, Non-Archimedian integrals and stringy Euler numbers of log terminal pairs, J. Eur. Math. Soc. (JEMS) 1 (1999), 5-33.

[BCHM10] C. Birkar, P. Cascini, C. Hacon, and J. McKernan, Existence of minimal models for varieties of log general type, J. Amer. Math. Soc. 23 (2010), no. 2, 405-468.

[dFKX12] T. de Fernex, J. Kollár, and C. Xu, The dual complex of singularities, Proceedings of the conference in honor of Yujiro Kawamata's 60th birthday, Advanced Studies in Pure Mathematics, 2012, arXiv:1212.1675.

[DL99] J. Denef and F. Loeser, Motivic Igusa zeta functions, J. Algebraic Geom. 7 (1998), 505-537.

[DL98] Germs of arcs on singular algebraic varieties and motivic integration, Invent. Math. 135 (1999), 201-232.

[DL02] Lefschetz numbers of iterates of the monodromy and truncated arcs, Topology 41 (2002), no. 5, 1031-1040.

[HN11] L. H. Halle and J. Nicaise, Motivic zeta functions of Abelian varieties, and the monodromy conjecture, Adv. Math. 227 (2011), 610-653.

[HN12] Motivic zeta functions for degenerations of Abelian varieties and Calabi-Yau varieties, Zeta functions in algebra and geometry (A. Campillo et al., eds.), Contemporary Mathematics, 566, pp. 233-259, American Mathematical Society, Providence, 2012.

[HX13] C. Hacon and C. Xu, Existence of log canonical closures, Invent. Math. 192 (2013), no. 1, 161-195.

[Kol13] J. Kollár, Singularities of the minimal model program, Cambridge Tracts in Math., 200, Cambridge University Press, Cambridge, 2013, with a collaboration of Sándor Kovács.

[KM98] J. Kollár and S. Mori, Birational geometry of algebraic varieties, Cambridge Tracts in Math., 134, Cambridge University Press, Cambridge, 1998, with the collaboration of C. H. Clemens and A. Corti; translated from the 1998 Japanese original.

[NS07] J. Nicaise and J. Sebag, Motivic Serre invariants, ramification, and the analytic Milnor fiber, Invent. Math. 168 (2007), no. 1, 133-173. 
[OX12] Y. Odaka and C. Xu, Log-canonical models of singular pairs and its applications, Math. Res. Lett. 19 (2012), 325-334.

[Vey03] W. Veys, Stringy zeta functions for $\mathbb{Q}$-Gorenstein varieties, Duke Math. J. 120 (2003), no. 3, 469-514.

Beijing International Center for Mathematical Research

Beijing 100871

China

cyxu@math.pku.edu.cn 